\title{
МАГНИТНО-РЕЗОНАНСНАЯ ТОМОГРАФИЯ ДЛЯ ПЕРСОНАЛИЗИРОВАННОЙ ОЦЕНКИ И ПРОГНОЗИРОВАНИЯ ЭФФЕКТИВНОСТИ ДОСТАВКИ НАНОФОРМУЛЯЦИЙ ПРОТИВООПУХОЛЕВЫХ ПРЕПАРАТОВ
}

В. А. Науменко ${ }^{1 凶}$, А. С. Гаранина ${ }^{1,2}$, С. С. Водопьянов ${ }^{1}$, А. А. Никитин ${ }^{1,2}$, А. О. Преловская ${ }^{1}$, Е. И. Демихов ${ }^{4}$ М. А. Абакумов ${ }^{1,3}$, А. Г. Мажуга ${ }^{1,2,5}$, В. П. Чехонин ${ }^{3}$

1 Лаборатория биомедицинских наноматериалов, Национальный исследовательский технологический университет «МИСиС», Москва

2 Научно-исследовательская лаборатория тканеспецифических лигандов, Химический факультет,

Московский государственный университет имени М. В. Ломоносова, Москва

${ }^{3}$ Российский национальный исследовательский медицинский университет имени Н. И. Пирогова, Москва

${ }^{4}$ Физический институт имени П. Н. Лебедева Российской академии наук, Москва

${ }^{5}$ Российский химико-технологический университет имени Д. И. Менделеева, Москва

\begin{abstract}
Магнитно-резонансная томография (МРТ) широко используется для диагностики онкологических заболеваний, а также для исследования доставки препаратов на магнитных наноносителях. Накопление наночастиц в опухоли высоко вариабельно в популяции и зависит от биологических факторов, которые во многом остаются неизученными. В последние годы было высказано предположение о возможности использования МРТ для предсказания ответа на терапию наноформулированными препаратами на основе скрининговых данных о накоплении в опухоли магнитноконтрастных диагностикумов. Несмотря на то что пилотные испытания указывают на принципиальную возможность предложенного подхода, существует ряд концептуальных проблем и технических ограничений для внедрения технологии в клинику. В статье обсуждаются преимущества и недостатки методов, позволяющих стратифицировать опухоли по степени накопления наночастиц. Дальнейшие исследования в данной области позволят разработать эффективные алгоритмы индивидуального лечения противоопухолевыми препаратами, доставляемыми на наночастицах.
\end{abstract}

Ключевые слова: противоопухолевая терапия, магнитно-резонансная томография, наночастицы, персонализированная медицина

Финансирование: работа выполнена при финансовой поддержке Министерства образования и науки РФ в рамках ФЦП «Исследования и разработки по приоритетным направлениям развития научно-технологического комплекса России на 2014-2020 годы», соглашение от 27.09.2017 г. № 14.575.21.0147 (уникальный идентисикатор соглашения RFMEFI57517X0147).

$\triangle$ Для корреспонденции: Виктор Алексеевич Науменко

Ленинский проспект, д. 4, г. Москва, 119049; naumenko.vict@gmail.com

Статья получена: 30.08.2018 Статья принята к печати: 25.09.2018

DOI: $10.24075 /$ vrgmu.2018.086

\section{MAGNETIC RESONANCE IMAGING FOR PREDICTING PERSONALIZED ANTITUMOR NANOMEDICINE EFFICACY}

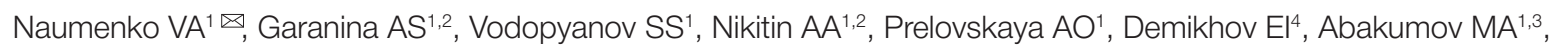
Majouga $A G^{1,2,5}$, Chekhonin VP3

${ }^{1}$ Biomedical Nanomaterials Laboratory, National University of Science and Technology MISIS, Moscow

Tissue-specific Ligands Research Laboratory, Faculty of Chemistry,

Lomonosov Moscow State University, Moscow

${ }^{3}$ Pirogov Russian National Research Medical University, Moscow

${ }^{4}$ Lebedev Physical Institute, Russian Academy of Sciences, Moscow

${ }^{5}$ Mendeleyev University of Chemical Technology of Russia, Moscow

Magnetic resonance imaging (MRI) is widely used to diagnose cancer and study patterns and effectiveness of nanocarrier delivery of anticancer drugs. Accumulation of nanoparticles in a tumor varies widely in a given population; it is also highly dependent on biological factors, which remain largely unstudied. In recent years, there was developed a hypothesis that suggests that MRI can be used to predict response to nanoformulations-based anticancer therapy since it provides data on accumulation of MRI contrast agents in the tumor. Pilot tests prove feasibility of the approach based on this hypothesis, however, there is a number of conceptual and technical problems and limitations that hamper its introduction into the routine clinical practice. This article discusses the advantages and disadvantages of methods to stratify tumors by level of nanoparticles accumulation. Further research in this field would facilitate development of effective algorithms of personalized treatment with anticancer drugs delivered by nanoparticles.

Keywords: anticancer therapy, magnetic resonance imaging, nanoparticles, personalized medicine

Funding: the study was financially supported by the Ministry of Education and Science of the Russian Federation under the Federal Targeted Programme for Research and Development in Priority Areas of Development of the Russian Scientific and Technological Complex for 2014-2020, Agreement \#14.575.21.0147 of 27.09.2017 (Agreement ID RFMEFI57517X0147)

Correspondence should be addressed: Victor A. Naumenko

Leninsky 4, Moscow, 119049; naumenko.vict@gmail.com

Received: 30.08.2018 Accepted: 25.09.2018

DOI: $10.24075 / \mathrm{brsmu} .2018 .086$ 
Онкологические заболевания являются одной из основных причин смертности, потери трудоспособности, снижения качества жизни пациентов и сопряженных с этим экономических потерь. Низкая эффективность традиционных методов лечения злокачественных новообразований (радикальная операция, химио- и лучевая терапия) диктует необходимость поисков новых подходов к решению проблемы. Основными мировыми трендами в области диагностики и лечения злокачественных новообразований являются: 1) внедрение высокоинформативных методов диагностики онкопатологии; 2) разработка новых препаратов и способов их доставки в опухоль; 3) переход к персонализированной медицине.

Использование наночастиц (НЧ) представляется перспективным во всех перечисленных концепциях [1]. Вопервых, магнитные НЧ (МНЧ) успешно используются для диагностики опухолевых заболеваний методом магнитнорезонансной томографии (МРТ). Во-вторых, уже одобрены к клиническому использованию несколько наноформуляций для доставки в опухоль химиотерапевтических препаратов (липосомы, полимерные мицеллы, НЧ на основе альбумина). На пересечении диагностического и терапевтического потенциала НЧ возникает возможность использования их для предсказания эффективности лечения у отдельных пациентов. Основная идея применения лабораторноинструментальных методов исследования в алгоритме персонализированной терапии наноформулированными противоопухолевыми препаратами (НПП) представлена на рис. 1. Для принятия решения о тактике лечения предлагается проводить скрининг опухолей на эффективность накопления наноносителей. В зависимости от результатов исследования (например, оценки степени накопления магнитного диагностикума в опухоли методом МРТ) можно составить прогноз накопления терапевтического препарата. Гипотеза заключается в том, что опухоли с эффективным накоплением контрастного диагностикума дадут более выраженный ответ на терапию нанопрепаратом.

Необходимость индивидуального прогноза обусловлена несколькими причинами. Во-первых, с точки зрения эффективности лечения, НПП следует назначать тогда, когда EPR-эффект (повышенная проницаемость кровеносных сосудов и сниженный лимфатический дренаж) обеспечивает аккумуляцию необходимой концентрации действующего вещества в опухоли. В противном случае перед назначением НПП необходимо использовать терапевтические стратегии, повышающие проницаемость опухолевых сосудов, например местную вазодилатацию путем нагревания, введения оксида азота, простагландинов (рис. 1). Доставка в опухоль может быть улучшена путем повышения артериального давления при введении ангиотензина II или коллагеназа-опосредованного разрушения опухолевого матрикса [2]. Наконец, недостаточность пассивного накопления препарата в опухоли можно преодолеть за счет использования НЧ с адресной доставкой [3, 4]. Во-вторых, с фармаэкономической точки зрения, при одинаковой эффективности противоопухолевого препарата в наноформуляции и без нее предпочтение должно отдаваться последнему, так как его себестоимость на несколько порядков ниже (например, стоимость 20 мг доксорубицина составляет 540 р., доксила - 42300 р.).

\section{Исследование прогностических факторов эффективности доставки противоопухолевых препаратов}

На сегодняшний день в мировой практике отсутствуют клинические алгоритмы индивидуальной оценки EPRэффекта и связанной с ним эффективности применения НПП. Однако в последние годы за рубежом запущены доклинические и клинические испытания, направленные на решение указанной проблемы.

В одной из работ на животной модели оценивали возможность использования магнитных частиц (ферумокситола) для определения эффективности лечения наноформулированным паклитакселом. После разделения животных на группы по степени выраженности EPR-эффекта на основании МРТ были показаны убедительные различия в уровне гибели опухолевых клеток и эффективности ответа на терапию в указанных группах [5]. В 2017 г. вышли первые результаты клинического исследования, в котором для оценки эффективности наноформулированного иринотекана у 13 пациентов с солидными опухолями использовали МРТ-данные о доставке в опухоль МНЧ

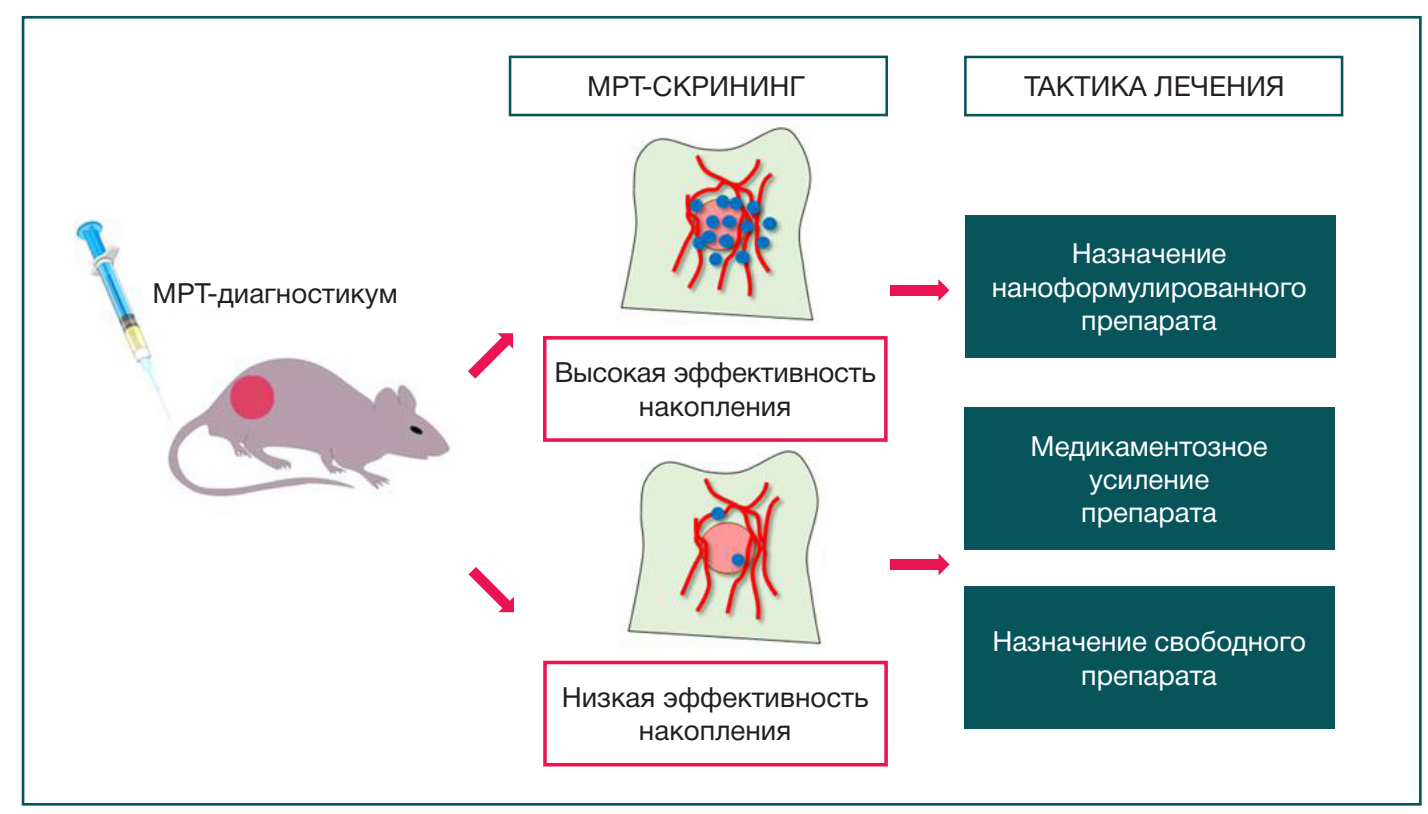

Рис. 1. Алгоритм персонализированной оценки и прогнозирования эффективности доставки НПП на животной модели 
(ферумокситола). Было показано, что высокие уровни накопления ферумокситола через 1-24 ч после введения коррелировали с уменьшением опухолевых очагов на фоне терапии [6]. Недостатком предложенного подхода является значительная разница в физических свойствах диагностических и терапевтических НЧ (в частности, их размер составлял 23 нм и 110 нм соответственно). Хорошо известно, что доставка НЧ в опухоль зависит от их гидродинамического размера: чем меньше частицы, тем эффективнее их экстравазация и перфузия в опухоли [7]. Проведение интравитальной микроскопии (ИВМ) для оценки фармакокинетики ферумокситола и PGLA-ПЭГ показало различия в скорости и локусах накопления двух типов НЧ с различным размером [5].

Прогноз эфффективности лечения липосомным доксорубицином рака молочной железы у крыс оценивали путем маммографии после введения иодинсодержащих липосом размером 100 нм [8]. На основании данных о накоплении контрастного вещества в опухоли удалось выделить группу животных с хорошим прогнозом лечения, что подтвердилось впоследствии. К сожалению, эффективность данного подхода была показана только на одной опухолевой модели. Предложенный метод основан на использовании рентгенологического исследования, уступающего по своей чувствительности и безопасности МРТ, что ограничивает перспективы его внедрения в клиническую практику.

Представляют интерес результаты клинического исследования, В котором оценивали корреляцию

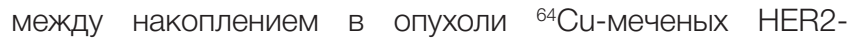
направленных ПЭГ-модифицированных липосом, содержащих доксорубицин, и эффективностью терапии у 19 пациентов с HER2-позитивным метастатическим раком молочной железы. Детекцию радиоактивно меченных Н4 проводили методом позитронно-эмиссионной и компьютерной томографии. Высокий уровень накопления меченых частиц коррелировал с положительным ответом на терапию [9]. Дизайн данного исследования был первоначально направлен на решение другой задачи оценку эффективности лечения рака молочной железы липосомным доксорубицином с трастузумабом в сочетании с циклосросфамидом. В связи с этим представляется затруднительной интерпретация вклада сопутствующих факторов в результаты эксперимента. Кроме того, объективно оценить применимость подхода не позволяло испытание лишь одной опухолевой модели.

Сходные данные получены в серии исследований с использованием наноформуляций доксорубицина, меченных радиоактивным технецием. На животной модели была показана корреляция интенсивности сигнала в опухоли по данным односотонной эмиссионной компьютерной томограсии с накоплением препарата в экстрагированных опухолях [10]. Эффективность данного подхода нашла подтверждение в клиническом испытании, где ${ }^{(99 m)}$ Тс-меченый липосомный доксорубицин вводили 35 пациентам с мезотелиомой. Накопление препарата в опухоли коррелировало с ответом на лечение [11]. Однако технические сложности, связанные с использованием радиоактивных материалов, а также отсутствие необходимого оснащения в большинстве лечебно-профилактических учреждений ограничивают трансляционный потенциал данного подхода.

Для оценки EPR-эффекта наряду с использованием методов in vivo визуализации предложены генные, белковые и клеточные прогностические маркеры. Например,

накопление липосом можно предсказать на основе соотношения металлопротеиназы 9 (MMP9) к тканевому ингибитору металлопротеиназы 1 (TIMP1) [12, 13]. Кроме того, исследуют возможность использования факторов роста эндотелиальных клеток (VEGFA) и фибробластов (FGF2), интерлейкинов (IL6, IL8), пептидов (эндостатин), а также клеток (эндотелиальных и их предшественников) в качестве маркеров EPR-эффекта $[14,15]$.

\section{Проблемы и перспективы использования МРТ в прогностических алгоритмах противоопухолевой терапии}

Наиболее перспективными нам представляются прогностические подходы, основанные на in vivo визуализации, которые были выполнены на животных моделях и вошли в стадию клинических испытаний. В отличие от анализа биомаркеров, в этих неинвазивных методах используют имеющиеся в лечебно-профилактических учреждениях оборудование и контрастные диагностикумы. K тому же МРТ обладает преимуществом перед радиоактивными и рентгенологическими методами оценки EPR-эффекта в силу своей безопасности и доступности. Однако существует ряд концептуальных проблем и технических ограничений, стоящих на пути создания технологии персонализированной оценки и прогнозирования эффективности доставки НПП:

До введения
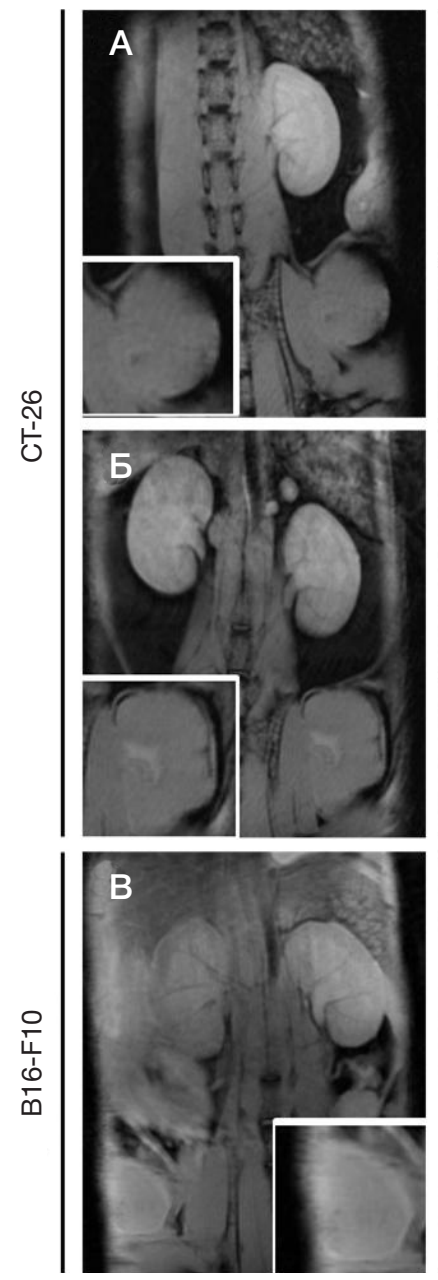

Рис. 2. Гетерогенность EPR-эффекта: внутригрупповая (А-Б); между разными моделями опухолей (A-B) 
1) в качестве диагностических и терапевтических препаратов используют НЧ различной природы [5];

2) не исследована возможность того, что введение первой (диагностической) дозы наноформуляции может оказывать влияние на введение второй (терапевтической) дозы;

3) не исследованы временные изменения EPR-эффекта в одной и той же опухоли, что может определять различия в накоплении первой и второй дозы НЧ;

4) в большинстве случаев исследования выполнены ретроспективно на небольшой выборке с использованием одного типа опухоли;

5) исследования не связаны с прямой оценкой EPRэффекта, а потому не позволяют исключить влияние сопутствующих факторов (например, сопутствующей противоопухолевой терапии).

Для всесторонней оценки гетерогенности EPR-эффекта и факторов, ее определяющих, необходимо исследование разных моделей (аллографтных и ксенографтных, ортотопических и гетеротопических) и типов опухолей Внутригрупповая неоднородность может быть оценена путем анализа различий в накоплении частиц у разных животных в рамках одной опухолевой модели. Исследования, выполненные в нашей лаборатории с использованием магнитно-контрастных НЧ, показали, что МРТ может быть использована для оценки степени выраженности EPRэффекта как для различных опухолевых моделей, так и для различных животных (рис. 2). На основе полученных данных возможно ранжирование животных на прогностические группы с последующей оценкой терапевтической эсффективности нанопрепаратов. Неоднородность накопления HЧ также может быть связана с эволюцией опухолевых сосудов и изменением архитектоники тканей, что диктует необходимость оценки EPR-эфффекта на разных этапах канцерогенеза.
Важным этапом внедрения в клинику индивидуального подхода к терапии нанопрепаратами является сравнение накопления первой и второй дозы НЧ. Во-первых, должны совпадать физико-химические свойства диагностических и терапевтических НЧ. Во-вторых, нельзя исключить, что введение первой дозы может оказывать влияние на последующие введения НЧ, например, за счет активации их захвата моноцитами/макрофагами - феномена, показанного ранее для последовательного введения онколитических вирусов. Наконец, описана возможность транзиторных изменений EPR-эффекта в одной и той же опухоли, что необходимо учитывать при оценке предсказательной силы первой дозы. Для моделирования биораспределения двух доз могут быть использованы НЧ, конъюгированные с разными красителями. ИВМ позволит оценить экстравазацию, диффузию, динамику накопления и клеточные мишени первой и второй доз в опухолевом микроокружении. Перспективным представляется также последовательное использование МРТ и ИВМ. Первый метод позволит осуществить скрининг опухолей с высоким и низким уровнями накопления НЧ, тогда как второй позволит исследовать клеточные механизмы, лежащие в основе различий EPR-эфффекта.

\section{ВЫВОДЫ}

Концепция использования неинвазивных методов и МНЧ для создания индивидуальных терапевтических алгоритмов в онкологии представляется перспективной и реалистичной. Использование современных методов исследования механизмов, определяющих степень накопления НЧ в опухоли, а также валидация МРТ как скринингового метода на животных моделях послужат основой для последующего внедрения в клиниескую практику технологии персонализированной оценки и прогнозирования эффективности доставки НПП.

\section{Литература}

1. Shi $J$ et al. Cancer nanomedicine: progress, challenges and opportunities. Nat Rev Cancer. NIH Public Access, 2017; 17 (1): 20-37.

2. Prabhakar $U$ et al. Challenges and key considerations of the enhanced permeability and retention effect for nanomedicine drug delivery in oncology. Cancer Res. 2013; 73 (8): 2412-17.

3. Davis ME et al. Evidence of RNAi in humans from systemically administered siRNA via targeted nanoparticles. Nature. 2010; 464 (7291): 1067-70.

4. Hrkach $\mathrm{J}$ et al. Preclinical development and clinical translation of a PSMA-targeted docetaxel nanoparticle with a differentiated pharmacological profile. Sci Transl Med. 2012; 4 (128): 128 ra39.

5. Miller MA et al. Predicting therapeutic nanomedicine efficacy using a companion magnetic resonance imaging nanoparticle. Sci Transl Med. 2015; 7 (314): 314ra183.

6. Ramanathan RK et al. Correlation between Ferumoxytol Uptake in Tumor Lesions by MRI and Response to Nanoliposomal Irinotecan in Patients with Advanced Solid Tumors: A Pilot Study. Clin Cancer Res. 2017; 23 (14): 3638-48.

7. Wilhelm $\mathrm{S}$ et al. Analysis of nanoparticle delivery to tumours. Nat Rev Mater. 2016; 1 (5): 16014.

8. Karathanasis $\mathrm{E}$ et al. Imaging nanoprobe for prediction of outcome of nanoparticle chemotherapy by using mammography. Radiology. 2009; 250 (2): 398-406.

9. Lee $\mathrm{H}$ et al. 64Cu-MM-302 Positron Emission Tomography Quantifies Variability of Enhanced Permeability and Retention of Nanoparticles in Relation to Treatment Response in Patients with Metastatic Breast Cancer. Clin Cancer Res. 2017; 23 (15): 4190-02.

10. Head HW et al. Combination radiofrequency ablation and intravenous radiolabeled liposomal Doxorubicin: imaging and quantification of increased drug delivery to tumors. Radiology. 2010; 255 (2): 405-14.

11. Arrieta $O$ et al. A phase II trial of prolonged, continuous infusion of low-dose gemcitabine plus cisplatin in patients with advanced malignant pleural mesothelioma. Cancer Chemother Pharmacol. 2014; 73 (5): 975-82.

12. Yokoi $\mathrm{K}$ et al. Capillary-Wall Collagen as a Biophysical Marker of Nanotherapeutic Permeability into the Tumor Microenvironment. Cancer Res. 2014; 74 (16): 4239-46.

13. Yokoi $\mathrm{K}$ et al. Serum biomarkers for personalization of nanotherapeutics-based therapy in different tumor and organ microenvironments. Cancer Lett. 2014; 345 (1): 48-55.

14. Sessa $\mathrm{C}$ et al. Biomarkers of angiogenesis for the development of antiangiogenic therapies in oncology: tools or decorations? Nat Clin Pract Oncol. 2008; 5 (7): 378-91.

15. Sherwood LM, Parris EE, Folkman J. Tumor Angiogenesis: Therapeutic Implications. N Engl J Med. 1971; 285 (21): 1182-6. 


\section{References}

1. Shi $J$ et al. Cancer nanomedicine: progress, challenges and opportunities. Nat Rev Cancer. NIH Public Access, 2017; 17 (1): 20-37.

2. Prabhakar $U$ et al. Challenges and key considerations of the enhanced permeability and retention effect for nanomedicine drug delivery in oncology. Cancer Res. 2013; 73 (8): 2412-17.

3. Davis ME et al. Evidence of RNAi in humans from systemically administered siRNA via targeted nanoparticles. Nature. 2010; 464 (7291): 1067-70.

4. Hrkach $\mathrm{J}$ et al. Preclinical development and clinical translation of a PSMA-targeted docetaxel nanoparticle with a differentiated pharmacological profile. Sci Transl Med. 2012; 4 (128): 128ra39.

5. Miller MA et al. Predicting therapeutic nanomedicine efficacy using a companion magnetic resonance imaging nanoparticle. Sci Transl Med. 2015; 7 (314): 314ra183.

6. Ramanathan RK et al. Correlation between Ferumoxytol Uptake in Tumor Lesions by MRI and Response to Nanoliposomal Irinotecan in Patients with Advanced Solid Tumors: A Pilot Study. Clin Cancer Res. 2017; 23 (14): 3638-48.

7. Wilhelm S et al. Analysis of nanoparticle delivery to tumours. Nat Rev Mater. 2016; 1 (5): 16014

8. Karathanasis $\mathrm{E}$ et al. Imaging nanoprobe for prediction of outcome of nanoparticle chemotherapy by using mammography. Radiology. 2009; 250 (2): 398-406.
9. Lee $\mathrm{H}$ et al. 64Cu-MM-302 Positron Emission Tomography Quantifies Variability of Enhanced Permeability and Retention of Nanoparticles in Relation to Treatment Response in Patients with Metastatic Breast Cancer. Clin Cancer Res. 2017; 23 (15): 4190-02.

10. Head HW et al. Combination radiofrequency ablation and intravenous radiolabeled liposomal Doxorubicin: imaging and quantification of increased drug delivery to tumors. Radiology. 2010; 255 (2): 405-14

11. Arrieta $\mathrm{O}$ et al. A phase II trial of prolonged, continuous infusion of low-dose gemcitabine plus cisplatin in patients with advanced malignant pleural mesothelioma. Cancer Chemother Pharmacol. 2014; 73 (5): 975-82.

12. Yokoi $\mathrm{K}$ et al. Capillary-Wall Collagen as a Biophysical Marker of Nanotherapeutic Permeability into the Tumor Microenvironment. Cancer Res. 2014; 74 (16): 4239-46.

13. Yokoi $K$ et al. Serum biomarkers for personalization of nanotherapeutics-based therapy in different tumor and organ microenvironments. Cancer Lett. 2014; 345 (1): 48-55.

14. Sessa $\mathrm{C}$ et al. Biomarkers of angiogenesis for the development of antiangiogenic therapies in oncology: tools or decorations? Nat Clin Pract Oncol. 2008; 5 (7): 378-91.

15. Sherwood LM, Parris EE, Folkman J. Tumor Angiogenesis: Therapeutic Implications. N Engl J Med. 1971; 285 (21): 1182-6. 